\title{
Aortic graft perforation by a rib stump after thoracic aorta replacement: A case report and literature review
}

Tomoko Ishii, MD, ${ }^{\mathrm{a}}$ Yusuke Iguchi, MD, ${ }^{\mathrm{a}}$ Toru Tsukada, MD, ${ }^{\mathrm{a}}$ and Motoo Osaka, MD, PhD, ${ }^{\mathrm{b}}$ Tsukuba, Japan

\footnotetext{
From the ${ }^{\mathrm{a}}$ Department of Cardiovascular Surgery, University of Tsukuba Hospital, Tsukuba, Japan; and ${ }^{\mathrm{b}}$ Faculty of Medicine, Department of Cardiovascular Surgery, University of Tsukuba, Tsukuba, Japan.

Disclosures: The authors reported no conflicts of interest.

The Journal policy requires editors and reviewers to disclose conflicts of interest and to decline handling or reviewing manuscripts for which they may have a conflict of interest. The editors and reviewers of this article have no conflicts of interest.

Received for publication March 10, 2021; accepted for publication March 10, 2021; available ahead of print March 13, 2021.

Address for reprints: Motoo Osaka, MD, PhD, Faculty of Medicine, Department of Cardiovascular Surgery, University of Tsukuba, 1-1-1 Tennodai, Tsukuba, 305-8575, Japan (E-mail: m-oosaka@md.tsukuba.ac.jp). JTCVS Techniques 2021;8:40-2

2666-2507

Copyright (C) 2021 The Author(s). Published by Elsevier Inc. on behalf of The American Association for Thoracic Surgery. This is an open access article under the CC BY-NC-ND license (http://creativecommons.org/licenses/bync-nd/4.0/).

https://doi.org/10.1016/j.xjtc.2021.03.015
}

Aortic graft perforation by a rib stump after thoracotomy is extremely rare. We present a case of fatal aortic graft perforation by sustained rib contact after descending aorta replacement for chronic type B aortic dissection with a review of the relevant literature.

\section{CASE DESCRIPTION}

A 54-year-old man was referred to our hospital with a chronic type B dissecting and descending aortic aneurysm, measuring $55 \mathrm{~mm}$ in diameter, that ranged from the left subclavian artery to the tenth thoracic vertebra. During surgery, the posterior portion of the left fourth rib was cut, and the fifth and sixth ribs were cut at the middle axillary line. The sixth intercostal space was then opened, each rib was stump smoothed with a file, and partial cardiopulmonary bypass was established before graft replacement (Triplex Straight, 26-mm Terumo Aortic; Vascutec, Inchinnan, Scotland) was performed. The chest was closed using rib pins for the fifth and sixth (but not the fourth) ribs and the graft alongside the aneurysmal wall was left unwrapped. Routine, evaluative computed tomography (CT) performed on postoperative day (POD) 12 revealed overlapping fourth rib stumps, one of which was protruding toward the chest cavity in contact with the aortic graft (Figure 1).

On POD 18, the patient suddenly collapsed. Cardiopulmonary resuscitation was started, and emergency CT revealed massive intrathoracic bleeding. A quick redo thoracotomy revealed a longitudinal 8-mm tear on the aortic prosthesis close to the fourth rib stump (Figure 2). Partial graft clamping and direct suturing of the tear successfully restored hemostasis, and all rib stumps were smoothed again before wrapping with felt strips. Despite our efforts, however, death occurred from hypoxic encephalopathy on POD 20. As Institutional Review Board approval was

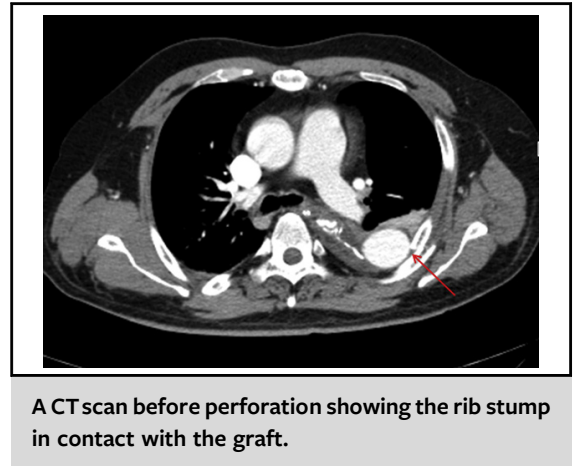

\begin{abstract}
CENTRAL MESSAGE
Aortic graft perforation due to sustained contact with a rib stump is a rare but fatal complication. Every effort should be made to avoid contact of vascular prostheses with hard, sharp tissues.
\end{abstract}

See Commentaries on pages 43 and 44 . waived owing to the single case report nature, individual informed consent was not required.

\section{DISCUSSION}

Only 3 cases of aortic graft perforation from rib stump friction after thoracotomy have been described since $2009,{ }^{1-3}$ in addition to 4 cases of graft perforation from other causes, such as sharp calcification inside the wrapped aortic wall, ${ }^{4,5}$ exposed normal ribs, ${ }^{6}$ or spinal bone spurs. ${ }^{7}$ Table 1 presents the demographics and clinical outcomes of 8 graft perforation cases caused by rib stumps or other factors, including the present case. ${ }^{1-7}$ All 4 rib stump graft perforations occurred after descending or thoracoabdominal aortic replacement for chronic aortic dissection, with an onset time ranging from 18 days to 6 months after surgery. Three patients with early onset developed left hemothorax, and 2 of these patients collapsed suddenly and died.

The larger size of the tears in rib stump cases are often thought to cause massive bleeding and collapse, but late 


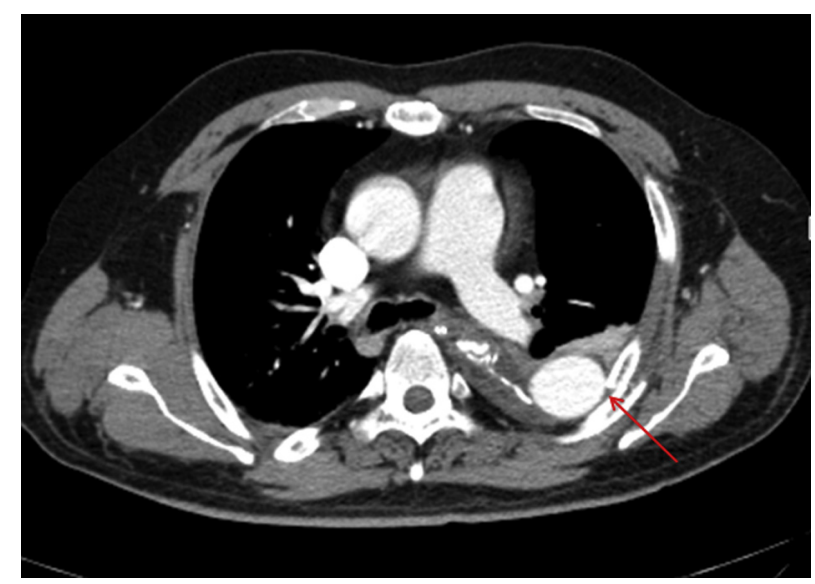

FIGURE 1. Computed tomography scan on postoperative day 12 after thoracic aortic replacement showing the fourth rib stump in contact with the aortic graft (see red arrow).

onset may localize bleeding by tissue adhesion, especially with smaller tears. In the present case, even after spotting rib stump contact on POD 12, we could not anticipate possible graft disruption by sustained friction because the rib edges had been carefully smoothed. Given that this unfortunate and lethal complication is rarely reported, we believe that extra caution is warranted whenever the possibility of rib stump tearing is encountered.

Tanaka and colleagues ${ }^{4}$ experimentally investigated the durability of grafts against friction in a simulated calcified aorta model. Their ex vivo experiment, consisting of an acryl tube containing fragments of shell, disrupted 2 of 3 adjacent pulsatile Dacron grafts on the 10th and 18th days, similar in timing to our present case. Careful consideration thus should be given to avoid any rib stump-to-graft contact, together with removing hard and sharp objects around the graft to the furthest extent possible. If contact is unavoidable, a cushioning wrap around the graft by the aortic wall is mandatory, and if contact between rib stumps and vascular grafts is observed on postoperative CT, reoperation should be immediate.

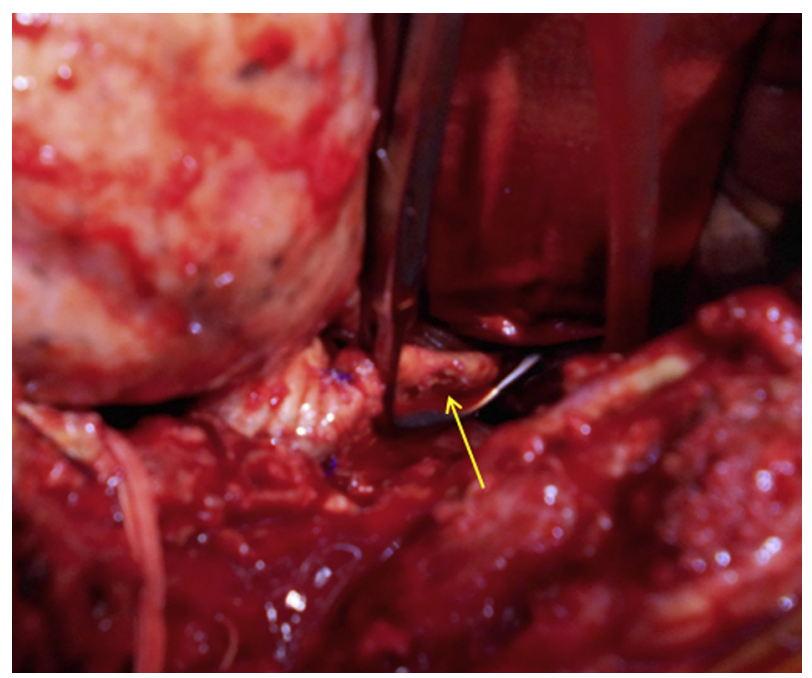

FIGURE 2. An 8-mm tear of the aortic graft was observed during the emergent chest reexploration for massive bleeding on postoperative day 18 (see yellow arrow).

\section{CONCLUSIONS}

We experienced a rare and fatal case of aortic graft perforation due to sustained friction with a rib stump. Contact of vascular prostheses with hard, sharp tissues should be avoided.

\section{References}

1. Yamamoto H, Yamamoto F, Ishibashi K, Noishiki Y. Vascular prosthesis rupture caused by contact with rib stump after thoracic aorta replacement. J Vasc Surg. 2009;50:195-7.

2. Tshomba Y, Bertoglio L, Marone EM, Psacharopulo D, Iob G, Chiesa R. Graft perforation by a dislocated rib fracture after open thoracoabdominal aortic repair: emergent endovascular and open repair. J Endovasc Ther. 2010;17:126-30.

3. Yalcin M, Aytekin I. An unusual complication: aortic graft perforation by a fractured rib after type B aortic dissection. Interact Cardiovasc Thorac Surg. 2016;23:338-9.

4. Tanaka H, Okada K, Yamashita T, Kawanishi Y, Matsumori M, Okita Y. Disruption of the vascular prosthesis caused by aortic calcification after replacement of the thoracoabdominal aortic aneurysm. Ann Thorac Surg. 2006;82:1097-9.

5. Ozaki N, Wakita N, Tanaka Y, Yuji D. The life-threatening complication of vascular graft rupture early after a thoracic aortic replacement. Eur J Cardiothorac Surg. 2012;42:737-8.

6. Matsuyama K, Kuinose M, Koizumi N, Iwahashi T, Toguchi K, Ogino H. A rare case of Dacron graft rupture due to friction against a rib. Thorac Cardiovasc Surg Rep. 2014;3:71-2.

7. Yoon SH, Park KH. Graft perforation by a spinal bony spur: an unusual cause of late bleeding after thoracoabdominal aorta replacement. Korean J Thorac Cardiovasc Surg. 2019;52:186-8. 
TABLE 1. Reported cases of aortic graft perforation by rib stumps and other causes

\begin{tabular}{|c|c|c|c|c|c|c|c|c|c|c|c|}
\hline Author & Year & $\begin{array}{c}\text { Age } \\
\text { and sex }\end{array}$ & Diagnosis & $\begin{array}{l}\text { Surgical } \\
\text { procedure }\end{array}$ & $\begin{array}{c}\text { Onset of } \\
\text { perforation }\end{array}$ & Symptoms & Imaging findings & $\begin{array}{c}\text { Surgical } \\
\text { intervention }\end{array}$ & $\begin{array}{l}\text { Length } \\
\text { of tear }\end{array}$ & $\begin{array}{c}\text { Cause of } \\
\text { perforation }\end{array}$ & Outcomes \\
\hline \multicolumn{12}{|l|}{$\begin{array}{l}\text { Due to rib } \\
\text { stump }\end{array}$} \\
\hline $\begin{array}{l}\text { Yamamoto } \\
{\text { et } \mathrm{al}^{1}}^{1}\end{array}$ & 2009 & $42 \mathrm{M}$ & $\begin{array}{l}\text { DTAA } \\
\text { (St A) }\end{array}$ & $\begin{array}{l}\text { TAR, } \\
\text { DAR }\end{array}$ & $25 \mathrm{~d}$ & $\begin{array}{l}\text { Sudden circulatory } \\
\text { collapse }\end{array}$ & $\begin{array}{l}\text { Massive left } \\
\text { hemothorax }\end{array}$ & SR & $6 \mathrm{~mm}$ & 8th rib stump & Died \\
\hline $\begin{array}{l}\text { Tshomba } \\
\text { et } \mathrm{al}^{2}\end{array}$ & 2010 & $40 \mathrm{~F}$ & $\begin{array}{l}\text { DTAAA } \\
\text { (Cf II) }\end{array}$ & TAAR & $6 \mathrm{mo}$ & No symptoms & $\begin{array}{l}\text { Massive bleeding in } \\
\text { rib stump removal }\end{array}$ & $\begin{array}{l}\text { TEVAR } \\
\text { and SR }\end{array}$ & $\begin{array}{l}\text { Not } \\
\quad \text { described }\end{array}$ & 6th rib stump & Discharged \\
\hline $\begin{array}{r}\text { Yalcin and } \\
\text { Aytekin }^{3}\end{array}$ & 2016 & $49 \mathrm{M}$ & $\begin{array}{l}\text { DTAA } \\
\text { (St B) }\end{array}$ & DAR & $45 \mathrm{~d}$ & $\begin{array}{l}\text { Dyspnea, bulging } \\
\text { of thoracotomy }\end{array}$ & $\begin{array}{l}\text { Significant left } \\
\text { hemothorax }\end{array}$ & $\mathrm{SR}$ & $5-6 \mathrm{~mm}$ & 4th rib stump & Discharged \\
\hline $\begin{array}{l}\text { Present } \\
\text { case }\end{array}$ & & $54 \mathrm{M}$ & $\begin{array}{l}\text { DTAA } \\
\text { (St B) }\end{array}$ & DAR & $18 \mathrm{~d}$ & $\begin{array}{l}\text { Sudden circulatory } \\
\text { collapse }\end{array}$ & $\begin{array}{l}\text { Massive left } \\
\text { hemothorax }\end{array}$ & SR & $8 \mathrm{~mm}$ & 4th rib stump & Died \\
\hline \multicolumn{12}{|l|}{ Other causes } \\
\hline $\begin{array}{c}\text { Tanaka } \\
\text { et } \mathrm{al}^{4}\end{array}$ & 2006 & $52 \mathrm{M}$ & TAAA & TAAR & $18 \mathrm{~d}$ & $\begin{array}{l}\text { Sudden circulatory } \\
\text { collapse }\end{array}$ & $\begin{array}{l}\text { Massive left } \\
\text { hemothorax }\end{array}$ & SR & $1 \mathrm{~mm}$ & $\begin{array}{l}\text { Aortic } \\
\text { calcification }\end{array}$ & Discharged \\
\hline $\begin{array}{l}\text { Ozaki } \\
\text { et } \mathrm{al}^{5}\end{array}$ & 2012 & $74 \mathrm{M}$ & $\begin{array}{l}\text { DTAA } \\
\text { (St B) }\end{array}$ & DAR & $30 \mathrm{~d}$ & $\begin{array}{l}\text { Massive bloody } \\
\text { effusion }\end{array}$ & Perigraft extravasation & SR & Small hole & $\begin{array}{l}\text { Aortic } \\
\quad \text { calcification }\end{array}$ & Discharged \\
\hline $\begin{array}{l}\text { Matsuyama } \\
{\text { et } \mathrm{al}^{6}}\end{array}$ & 2014 & $54 \mathrm{M}$ & $\begin{array}{l}\text { AEF, } \\
\text { TAAA } \\
\text { (Cf III) }\end{array}$ & $\begin{array}{l}\text { DAR, } \\
\text { TAAR }\end{array}$ & $4 \mathrm{mo}$ & $\begin{array}{l}\text { Sudden back pain } \\
\text { and preshock }\end{array}$ & $\begin{array}{l}\text { Extravasation and } \\
\text { perigraft hematoma }\end{array}$ & $\begin{array}{l}\text { TEVAR } \\
\text { and SR }\end{array}$ & Small hole & $\begin{array}{l}\text { Normal uncut } \\
\text { 10th rib }\end{array}$ & Discharged \\
\hline $\begin{array}{l}\text { Yoon and } \\
\text { Park }^{7}\end{array}$ & 2019 & $78 \mathrm{M}$ & $\begin{array}{l}\text { TAAA } \\
\text { (Cf III) }\end{array}$ & TAAR & $49 d$ & $\begin{array}{l}\text { Fever, abdominal } \\
\text { pain and distention }\end{array}$ & $\begin{array}{l}\text { Large retroperitoneal } \\
\text { hematoma }\end{array}$ & SR & $2 \mathrm{~mm}$ & $\begin{array}{l}\text { L2 spinal } \\
\text { bony spur }\end{array}$ & Discharged \\
\hline
\end{tabular}

$\overline{D T A A}$, Dissecting thoracic aortic aneurysm; $S t$, Stanford type; TAR, total arch replacement; $D A R$, descending aortic replacement; $S R$, surgical repair; $D T A A A$, dissecting thoracoabdominal aortic aneurysm; $C f$, Crawford type; TAAR, thoracoabdominal aortic replacement; TEVAR, thoracic endovascular aortic repair; TAAA, thoracoabdominal aortic aneurysm; $A E F$, aorto-esophageal fistula. 